\title{
Rational Decisions, Random Matrices and Spin Glasses
}

\author{
Stefano Galluccio ${ }^{\dagger}$, Jean-Philippe Bouchaud ${ }^{\dagger, *}$ and Marc Potters ${ }^{\dagger}$ \\ $\dagger$ Science 65 Finance, 109-111 rue Victor Hugo, 92523 Levallois Cedex, FRANCE \\ * Service de Physique de l'État Condensé, Centre d'études de Saclay, \\ Orme des Merisiers, 91191 Gif-sur-Yvette Cédex, FRANCE
}

(March 6, 2018)

\begin{abstract}
We consider the problem of rational decision making in the presence of nonlinear constraints. By using tools borrowed from spin glass and random matrix theory, we focus on the portfolio optimisation problem. We show that the number of "optimal" solutions is generically exponentially large: rationality is thus de facto of limited use. In addition, this problem is related to spin glasses with Lévy-like (long-ranged) couplings, for which we show that the ground state is not exponentially degenerate.
\end{abstract}

It is often hoped that science can help in choosing the right decision. A rational decision is usually thought of as the solution which optimizes a certain utility (or cost) function, which is supposed to quantify the performance of a given strategy. A simple example is that of portfolio optimization: given a set of financial assets, characterized by their average return and their risk, what is the optimal weight of each asset, such that the overall portfolio provides the best return for a fixed level of risk, or conversely, the smallest risk for a given overall return? It is frequently the case that the optimal solution is unique, so that 'rational' operators should choose this particular one, unless they are inefficient. In the case of portfolio selection, the only free parameter is the proportion of the "riskless asset"; all portfolios should thus look very much alike. This is at the heart of the so called Capital Asset Pricing Model, (CAPM), one of the cornerstones of modern theoretical finance [1].

In this Letter we will show that, surprisingly, there are cases where the number of "rational" decisions is exponentially large (for example in the number of assets) much like the number of ground states in spin glasses or other disordered systems [2]. This means that there is an irreducible component of randomness in the final decision: the degeneracy between this very large number of possibilities can only be lifted by small, 'irrational', effects.

More precisely, the average return $R_{P}$ of a portfolio $P$ of $N$ assets, is defined as $R_{P}=\sum_{i=1}^{N} p_{i} R_{i}$, where $p_{i}$ $(i=1, \cdots, N)$ is the amount of total capital invested in the asset $i$, and $\left\{R_{i}\right\}$ are the expected returns of the individual assets. Similarly, the risk on a portfolio can be associated to the total variance $\sigma_{P}^{2}=\sum_{i=1}^{N} p_{i}^{2} \sigma_{i}^{2}+$ $\sum_{i \neq j}^{N} p_{i} p_{j} \sigma_{i j}$, where $\sigma_{i}^{2}$ are the single asset variances and $\sigma_{i j}$ are the inter-asset covariances. Alternatively, one can define $\sigma_{P}^{2}=\sum_{i, j=1}^{N} p_{i} C_{i j} p_{j}$ where $C_{i j}$ is the covariance matrix.

Generally speaking, the goal of each rational investor consists in maximizing the portfolio return and/or in minimizing its variance (or risk). This optimization pro- cedure, or portfolio selection, as investigated in its simplest version by Markowitz [3], results in a set of all possible "optimal" couples $\left\{R_{P}, \sigma_{P}^{2}\right\}$ which all lie on a curve called the efficient frontier (EF). For a given degree of risk, there is thus a unique selection of assets $\left\{p_{i}^{*}\right\}_{i=1}^{N}$ which maximizes the expected return. On the opposite, we will show that in some cases this simple scenario does not hold: a very large number of compositions $\left\{p_{i}\right\}_{i=1}^{N}$ can be "optimal", in the sense defined above. Furthermore, these optimal compositions can be very different from another.

In order to illustrate this rather general scenario with an explicit example, we shall investigate the problem of portfolio selection in the case one can buy, but also to short sell stocks, currencies, commodities and other kinds of financial assets. This is the case of 'futures' markets or margin accounts. The only requirement is to leave a certain deposit (margin) proportional to the value of the underlying asset [4]. This means that the overall position on these markets is limited by a constraint of the form:

$$
\sum_{i=1}^{N} \gamma W_{i}\left|p_{i}\right|=\mathcal{W}
$$

where $W_{i}$ is the price of asset $i, \mathcal{W}$ the total wealth of the operator, and $\gamma$ the fraction defining the margin requirement. Here, $p_{i}$ is the number of contracts on asset $i$ which are bought $\left(p_{i}>0\right)$ or sold $\left(p_{i}<0\right)$. It is worth stressing that it is the nonlinear form of the above constraint that makes the problem interesting, as we shall see below. At this point we simply note that the optimal portfolio $p_{i}^{*}$, which - say - minimizes the risk for a given return, can be obtained using two Lagrange multipliers $(\nu, \mu)$ :

$$
\frac{\partial}{\partial p_{i}}\left[\frac{1}{2} \sum_{j, k=1}^{N} p_{j} C_{j k} p_{k}-\nu \sum_{j=1}^{N} p_{j} R_{j}-\mu \sum_{j=1}^{N} W_{j}\left|p_{j}\right|\right]=0 .
$$

Without loss of generality, one can always set $W_{i} \equiv 1$. Defining $S_{i}$ to be the sign of $p_{i}^{*}$, one gets: 


$$
p_{i}^{*}=\nu \sum_{j=1}^{N} C_{i j}^{-1} R_{j}+\mu \sum_{j=1}^{N} C_{i j}^{-1} S_{j},
$$

where $\mathbf{C}^{-1}$ is the matrix inverse of $\mathbf{C}$. Taking the sign of this last equation leads to an equation for the $S_{i}$ 's which is identical to those defining the locally stable configurations in a spin-glass [2]:

$$
S_{i}=\operatorname{sign}\left[H_{i}+\sum_{j=1}^{N} J_{i j} S_{j}\right],
$$

where $H_{i} \equiv \nu \sum_{j}^{N} C_{i j}^{-1} R_{j}$ and $J_{i j} \equiv \mu C_{i j}^{-1}$ are the analogue of the "local field" and of the spin interaction matrix, respectively. Once the $S_{i}$ 's are known, the $p_{i}^{*}$ are determined by Eq. (3); $\nu$ and $\mu$ are fixed, as usual, so as to satisfy the constraints. Let us consider, for simplicity, the case where one is interested in the minimum risk portfolio, which corresponds to $\nu=0$. [By analogy with spin glasses, the following results are not expected to change qualitatively if $\nu \neq 0$ [5]]. In this case, one sees that only $|\mu|$ is fixed by the constraint. Furthermore, the minimum risk is given by $\mathcal{R}=\mu^{2} \sum_{j, k}^{N} S_{j} C_{j k}^{-1} S_{k}$, which is the analogue of the energy of the spin glass. It is now well known that if $\mathbf{J}$ is a random matrix, the so called "TAP" equation (舟), defining the metastable states of a spin glass at zero temperature, generically has an exponentially large (in $N$ ) number of solutions [2]. Note that, as stated above, the multiplicity of solutions is a direct consequence of the nonlinear constraint (1).

In what respect can the correlation matrix $\mathbf{C}$ be considered as random? Here we take a step in complete analogy with the original Wigner and Dyson's idea of replacing the Hamiltonian of a deterministic but complex system by a random matrix [7]. More precisely, they proposed to study the properties of one generic member of a statistical ensemble [8] which shares the symmetries of the original Hamiltonian. Similarly, in the present situation, we would like to see $\mathbf{C}^{-1}$ as a random matrix whose elements are distributed according to a given ensemble, compatible with some general properties. In our case, for instance, we must select $\mathbf{C}^{-1}$ from an ensemble of positive definite matrices, as all the eigenvalues of the covariance matrix are $>0$. The choice of a suitable statistical ensemble is guided by the following observation. The (daily) fluctuations of the asset $i, \delta W_{i}$, can be decomposed as:

$$
\delta W_{i}=\sum_{\alpha=1}^{K} M_{i \alpha} \delta E_{\alpha}+\delta W_{i 0},
$$

where the $E_{\alpha}$ are $K$ independent factors which affects the assets differently, and $\delta W_{i 0}$ is the part of the fluctuation which is specific to asset $i$ (and thus independent of the $E_{\alpha}$ ). The $E_{\alpha}$ contain all information on how the stochastic evolution of a given asset is correlated to the others. The matrix $C_{i j}$ can thus be written as $\mathbf{C}=\mathbf{M}$ $\mathbf{M}^{T}+\mathbf{D}$, where $\mathbf{D}$ is a positive diagonal matrix, and $\mathbf{M}$ a $N \times K$ rectangular matrix. The above representation ensures that all eigenvalues of $\mathbf{C}$ are positive. In order to simplify the problem, we shall make in the following the assumption that $\mathbf{D}$ is proportional to the identity matrix and that the coefficients $M_{i \alpha}$ are completely random. For $K=N, \mathbf{C}$ is a member of the so-called Exponential Orthogonal Ensemble (EOE) [6], which is a maximum entropy (least information) ensemble. For any $N \times K$ matrix $\mathbf{M}$, (with $N \geq K$ ), the density of eigenvalues $\rho_{C}(\lambda)$ of $\mathbf{C}$ is exactly known in the limit $N \rightarrow \infty, K \rightarrow \infty$ and $Q=K / N$ fixed [9]. In the limit $Q=1$ (which we consider from now on) the normalized eigenvalue density of the (square) matrix $\mathbf{M}$ is the well known semi-circle law, from which the density of eigenvalues of $\mathbf{C}$ and $\mathbf{J}=\mu \mathbf{C}^{-1}$ are easily deduced. In particular, for the situation we are interested in, one finds

$$
\left.\left.\rho_{C}(\lambda)=\frac{1}{2 \pi \tau} \frac{\sqrt{\tau(4+a)-\lambda}}{\sqrt{\lambda-a \tau}}, \quad \lambda \in\right] a \tau,(4+a) \tau\right] .
$$

where $a$ measures the relative amplitude of the diagonal contribution $\mathbf{D}$ and $\tau$ the width of the distribution. The distribution of eigenvalues of $\mathbf{J}=\mu \mathbf{C}^{-1}, \rho_{J}(x)$, can be easily calculated from Eq.(6). Note that in the case where $a=0, \rho_{J}(x)$ has a power-law tail decaying as $\rho_{J}(x) \propto x^{-3 / 2}$. For finite $a$, however, the maximum eigenvalue of $\mathbf{J}$ is $\propto 1 / a$.

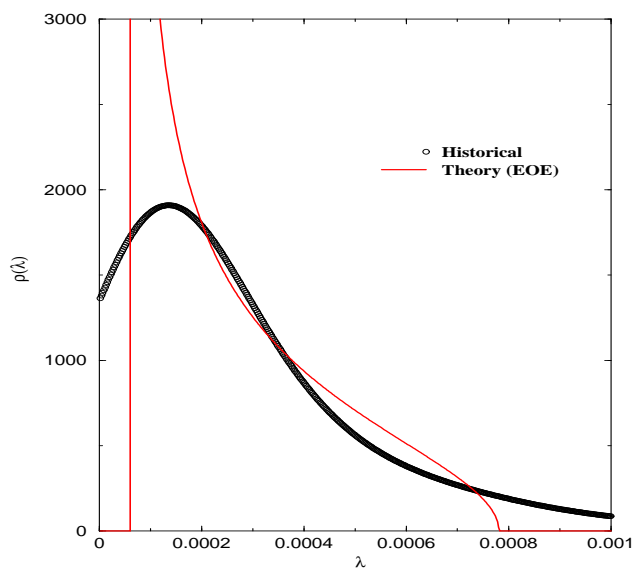

FIG. 1. Smoothed distribution of eigenvalues of $\mathbf{C}$, where the correlation matrix $\mathbf{C}$ is extracted from $N=406$ assets of the S\&P500 during the years 1991-1996. For comparison we have plotted the best fit obtained with the theoretical density (6) with $a \simeq .34, \tau \simeq 1.8 \times 10^{-4}$. Results are qualitatively similar in the case of the London or Zurich Stock Exchanges.

We have studied numerically the density of eigenvalues of the correlation matrix of 406 assets of the NewYork Stock Exchange (NYSE), based on daily variations during the years 1991-96, for a total of 1500 days. Moreover, by repeating our analysis on different Stock Markets (e.g. London and Zurich) we have checked the robustness 
and universality of our results. The observed density of eigenvalues is shown in Fig.1 together with the best fit obtained by assuming $Q=1$. The fitting parameters are $a$ and the width of the semi-circle distribution $\tau$, which are found to be, respectively, $a \simeq .34$ and $\tau \simeq 1.8 \times 10^{-4}$. The deviation from the theoretical curve is partially due to finite $N$ effects which are expected to smear out the singularities at $\lambda=a \tau$ and $(4+a) \tau$. It might also be that $\mathbf{D}$ has unequal diagonal elements (this would also smear out the singularities of $\rho_{J}(\lambda)$ ), or let the number $K$ of "explicative factors" be less than $N$ (a rather likely situation). Note that the empirical $\rho_{C}$ can be rather well fitted by a log-normal distribution, suggesting that the relevant ensemble has the additional symmetry $\mathbf{C} \leftrightarrow$ $\mathbf{C}^{-1}$, although we have not been able to justify this property.

The point, however, is not to claim that the density of eigenvalues is precisely described by the above model, but that it represents a reasonable approximation for our purposes. Once the density of eigenstates $\rho_{J}(\lambda)$ is known, the number of solutions of the optimization equation, averaged over the matrix ensemble, can be computed using tools borrowed from spin glasses and random matrix theory. As discussed in [10], the main ingredient is the generating function $G(x)$, defined as [11]:

$$
\int \mathcal{D}[\mathbf{J}] \exp \{\operatorname{Tr}(\mathbf{J A} / 2)\} \underset{N \gg 1}{\simeq} \exp \{N \operatorname{Tr} G(\mathbf{A} / N)\}
$$

where $\mathbf{A}$ is any symmetric $N \times N$ matrix of finite rank, and $\mathcal{D}[\mathbf{J}]=d[\mathbf{J}] P([\mathbf{J}])$ is the probability measure over the matrix ensemble. The above formula holds for general complex hermitian matrices $\mathbf{J}$ [11]. For example, in the simplest possible case, where $\mathbf{J}$ is extracted from a Gaussian Ensemble, one has $P([\mathbf{J}])=$ $\exp \left\{-\operatorname{Tr}\left(\mathbf{J}^{2} / 4 \sigma^{2}\right)\right\} / Z$ and thus $G(x)=x^{2} / 4$. Formally, $G(x)$ can be computed in general from $\rho_{J}(\lambda)$ using a series of rather involved transformations [12], which can be somewhat simplified by the use of a Hilbert integral transform of the eigenvalue density 13.

In the specific case (6), we have been able to compute $G_{a}(x)$ exactly for $a=0$, with the result $G_{0}(x)=-\sqrt{-x}$, and perturbatively in the limits $a \ll 1$ and $a \gg 1$. Leaving aside all mathematical details [13], the results are:

$$
\begin{aligned}
& G_{a}(x) \simeq \frac{x^{2}}{4 a^{4}}, \quad a \gg 1, \\
& G_{a}(x) \simeq-\sqrt{a} g\left(-\frac{x}{a}\right), \quad a \ll 1,
\end{aligned}
$$

where the scaling function $g(u)$ behaves as $g(u) \simeq \sqrt{u}$ for $u \gg 1$ and as $g(u) \propto u$ for $u \ll 1$. As a side remark, one can show that for Lévy random matrices [14] where $\rho(\lambda)$ decays as $\lambda^{-1-\beta}$ for large $\lambda$, the characteristic function behaves as $G(x) \simeq-(-x)^{\beta}$ for small $x$ 's, and for all $\beta<1$. Consequently, the problem we are concerned with here $(\beta=1 / 2)$ can be seen as the study of the metastable states in spin glasses with long-range interactions [15,13.
Once $G_{a}(x)$ is known, one can write the average number of solutions $\mathcal{N}$ of the TAP equations for large $N$ as $\mathcal{N} \sim \exp \{N f(a)\}$, where $f(a)$ is obtained from a steepest descent approximation. Indeed, from Eq.(4), one derives the following result, valid for large $N$ [10]:

$$
\begin{aligned}
\mathcal{N} & =\left\langle\sum_{\{S\}} \prod_{i=1}^{N} \theta\left(S_{i} \sum_{j=1}^{N} J_{i j} S_{j}\right)\right\rangle \\
& \simeq \max _{x, y, W, Z}\left\{\operatorname { e x p } \left[-x Z-y W+G_{a}(x+\sqrt{y})\right.\right. \\
& \left.\left.+G_{a}(x-\sqrt{y})+\ln \left(\operatorname{erfc}\left(-\frac{Z}{2 \sqrt{W}}\right)\right)\right]\right\} \\
& \equiv e^{N f(a)}
\end{aligned}
$$

where $\theta(u)$ is the usual Heavyside function of its argument. From (8), we find that $f(a)$ very rapidly converges to $\ln 2$ (the maximum allowed number of solutions) when $a$ is large:

$$
f(a)=\ln 2-e^{-a^{2} / 2}\left(\frac{1}{\sqrt{2 \pi} a}+o\left(\frac{1}{a^{2}}\right)\right),
$$

a result which we have numerically confirmed by extracting $\mathbf{J}$ from the proper statistical ensemble. To give an idea, one finds $f(a=1) \simeq .686 \pm .003$, which is already quite close to $\ln 2=.693147 \ldots$.

For small $a$, calculations become rather involved, as the extremizing set $\left\{x^{*}, y^{*}, W^{*}, Z^{*}\right\}$ corresponds to a minimum and actually lies on the domain's border. The final result is that $y^{*}=x^{* 2}, W^{*}=1 /\left(8 \sqrt{2 x^{* 3}}\right)$ and $Z^{*}=1 /\left(4 \sqrt{2 x^{*}}\right)$, which leads to $f(a) \propto a^{1 / 4}$ for $a \ll 1$. We have confirmed this result numerically (see Fig 2). Therefore, for $a=0, f(a=0)=0$, i.e. the number of metastable solutions does not grow exponentially with $N$. This result means that the presence of very large eigenvalues prevents the existence of many metastable states. This has an interesting consequence in the physical context, which was conjectured in 15: a spin glass with a broad distribution of couplings cannot sustain many ground states; one can thus expect that the low temperature phase of these models is rather different from the 'standard picture' 22. We can show that this is true for all $\beta<1[13]$; we find in particular that $f(a) \simeq K(\beta) a^{\beta / 2}$ for small $a$, with a prefactor $K(\beta)$ algebraically diverging for $\beta \rightarrow 1$. For $\beta>1$, finally, $f(a=0)>0$.

Let us now come back to our main theme. In what respect is the above picture a valid description of the portfolio optimization problem? In the real situation, of course, $\mathbf{C}$ is given by the "historical" correlation matrix. If our model is correct, then one should expect the number of optimal solutions to be exponentially large, even for small $a$ 's. By extracting true correlation matrices of sizes up to $N=20$ from the available 406 assets of the NYSE (see above), we have indeed numerically found that $\mathcal{N}_{\mathrm{NY}} \sim \exp \left(f_{\mathrm{NY}} N\right)$ with $f_{\mathrm{NY}}=.68 \pm .01$. This result 
does not qualitatively change in the case of the London or Zurich markets. Despite of the rather low dimensionality of the involved matrices, with unavoidable finite-size effects, the above result is quite well reproduced by our theoretical model (which is valid at $N \gg 1$ ). In fact, by using the fitting $(a, \tau)$ parameters as in Fig.1, we find a theoretical value $f_{\mathrm{TH}}=.63 \pm 0.02$, not too far from the real one. Similarly by extracting $\mathbf{C}$ from 172 stocks of the London Stock Exchange (LSE) we have found an empirical $f_{\mathrm{LSE}}=.43 \pm .09$ and a corresponding theoretical value $f_{\mathrm{TH}}=.32 \pm .07$. Remarkably, our admittedly approximate description of the density of states of the matrix $\mathbf{J}$ seems to account satisfactorily for the observed number of "optimal portfolios".

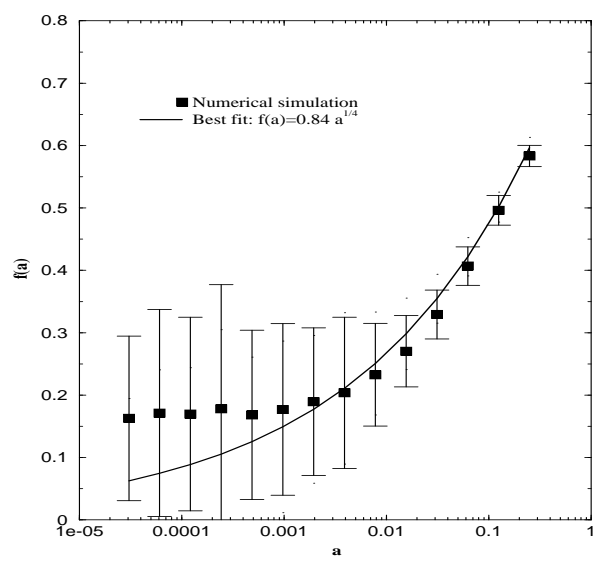

FIG. 2. The theoretical exponent $f(a)$ plotted vs $a(a \ll 1)$ when $\mathbf{C}$ is extracted from the EOE, using an exact enumeration procedure to find the numbers of solutions up to $N=27$. Finite size effects are clearly present for the smallest values of $a$. The theoretical line $K a^{1 / 4}$ is plotted for comparison, with $K \sim .84$.

By means of a combination of analytical and numerical arguments, we have thus shown that the number of optimal portfolios in futures markets (where the constraint on the weights is non linear) grows exponentially with the number of assets. On the example of U.S. stocks, we find that an optimal portfolio with 100 assets can be composed in $\sim 10^{29}$ different ways ! Of course, the above calculation counts all local optima, disregarding the associated value of the residual risk $\mathcal{R}$. One could extend the above calculations to obtain the number of solutions for a given $\mathcal{R}$. Again, in analogy with [10], we expect this number to grow as $\exp N f(\mathcal{R}, a)$, where $f(\mathcal{R}, a)$ has a certain parabolic shape, which goes to zero for a certain 'minimal' risk $\mathcal{R}^{*}$. But for any small interval around $\mathcal{R}^{*}$, there will already be an exponentially large number of solutions. The most interesting feature, with particular reference to applications in economy, finance or social sciences, is that all these quasi-degenerate solutions can be very far from each other: in other words, it can be rational to follow two totally different strategies!
Furthermore, these solutions are usually 'chaotic' in the sense that a small change of the matrix $\mathbf{J}$, or the addition of an extra asset, completely shuffles the order of the solutions (in terms of their risk). Some solutions might even disappear, while other appear. It is worth pointing out that the above scenario is not restricted to portfolio theory, but is germane to a variety of situations. For example, it is well known in Game Theory that each player has a different utility function he must maximize in order to get the best profit. When, in addition, nonlinear contraints are present, we expect a similar proliferation of solutions. As emphasized above, the existence of an exponentially large number of solutions forces one to rethink the very concept of rational decision making.

Aknowledgements: We want to thank J.P. Aguilar, R. Cont and L. Laloux for discussions. The LSE and Zurich data were obtained from the Financial Times and the NYSE from S\&P Compustat.

[1] E.J. Elton and M.J. Gruber, Modern Portfolio Theory and Investment Analysis (J.Wiley and Sons, New York, 1995).

[2] M. Mézard, G. Parisi and M.A. Visrasoro, Spin Glass Theory and Beyond (World Scientific, Singapore, 1987).

[3] H. Markowitz, Portfolio Selection: Efficient Diversification of Investments (J.Wiley and Sons, New York, 1959). See also: J.P. Bouchaud and M. Potters, Theory of Financial Risk, Aléa-Saclay, Eyrolles (Paris, 1997) (in french).

[4] For a recent account, see J. Hull, Futures, Options and Other Derivatives (Prentice Hall, New York, 1997).

[5] D.S. Dean, J. Phys. A. 27 L23, 1994

[6] B. V. Bronk, J. Math. Phys. 6, 228 (1965).

[7] For a review, see: O. Bohigas, M. J. Giannoni, Mathematical and computational methods in nuclear physics, Lecture Notes in Physics, Vol. 209, Springer-Verlag (1983)

[8] M. Mehta, Random Matrices (Academic Press, New York, 1995).

[9] A.M. Sengupta and P.P. Mitra Distribution of Singular Values for Some Random Matrices, cond-mat/9709283 preprint.

[10] G. Parisi and M. Potters, J. Phys. A, 28, 5267 (1995).

[11] C. Itzykson and J.-B. Zuber, J. Math. Phys. 21, 411 (1980).

[12] E. Marinari, G. Parisi and F. Ritort, J. Phys. A 277647 (1994).

[13] S. Galluccio, J.P. Bouchaud and M. Potters, unpublished.

[14] P. Cizeau and J.P. Bouchaud, Phys. Rev. E 501810 (1994).

[15] P. Cizeau and J.P. Bouchaud, J. Phys. A, 26 L187 (1993). 